\title{
A Counterexample to a Question of Hof, Knill and Simon
}

\author{
Sébastien Labbé* \\ LIAFA, Université Paris Diderot - Paris 7, \\ Case 7014, 75205 Paris Cedex 13, France \\ labbe@liafa.univ-paris-diderot.fr \\ Submitted: Sep 27, 2013; Accepted: Jul 9, 2014; Published: Jul 21, 2014 \\ Mathematics Subject Classifications: 68R15, 37B10
}

\begin{abstract}
In this article, we give a negative answer to a question of Hof, Knill and Simon (1995) concerning purely morphic sequences obtained from primitive morphism containing an infinite number of palindromes. Their conjecture states that such palindromic sequences arise from substitutions that are in class $\mathcal{P}$. The conjecture was proven for the binary alphabet by B. Tan in 2007 . We give here a counterexample for a ternary alphabet.
\end{abstract}

Keywords: Hof, Knill and Simon conjecture; class $\mathcal{P}$; palindromes; stabilizer.

\section{Introduction}

A morphism of monoid $\varphi: \mathcal{A}^{*} \rightarrow \mathcal{A}^{*}$ is in class $\mathcal{P}$ if there exists a palindrome $p$ and for every $\alpha \in \mathcal{A}$ there exists a palindrome $q_{\alpha}$ such that $\varphi(\alpha)=p q_{\alpha}$ [HKS95]. If $\mathbf{x}$ is an infinite sequence generated by a morphism in class $\mathcal{P}$, then Hof, Knill and Simon proved that $\mathbf{x}$ contains an infinite number of palindrome factors. Such morphisms allowed them to derive some discrete Schrödinger operators having purely singular continuous spectrum. For example, on the binary alphabet $\mathcal{A}=\{a, b\}$, the morphism $\rho: a \mapsto b b a b a, b \mapsto b b a$ is in class $\mathcal{P}$. The morphism $\theta: a \mapsto a b, b \mapsto b a$ is not in class $\mathcal{P}$, but its square $\theta^{2}: a \mapsto a b b a, b \mapsto b a a b$ is in class $\mathcal{P}$. Therefore, the fixed point

$$
\mathbf{x}_{\rho}=\rho\left(\mathbf{x}_{\rho}\right)=\text { bbabbabbababbabbabbababbabbabbababbabbab } \cdots
$$

and the fixed point, also known as the Thue-Morse word,

$$
\mathbf{x}_{\theta}=\theta\left(\mathbf{x}_{\theta}\right)=a b b a b a a b b a a b a b b a b a a b a b b a a b b a b a a b b a a b a b b a \cdots
$$

\footnotetext{
*With the support of NSERC (Canada)
} 
are both palindromic, i.e., both contain an infinite number of palindromes. The question of Hof, Knill and Simon that motivates this article concerns the reciprocal. In their article, they wrote "Clearly, we could include into class $\mathcal{P}$ substitutions of the form $s(b)=q_{b} p$. We do not know whether all palindromic $x_{s}$ arise from substitutions that are in this extended class $\mathcal{P}$." It turns out that we need to extend a little bit more the class $\mathcal{P}$ by including also the conjugates. Blondin Massé (proof available in [Lab08, Prop 3.5]) proved that the primitive morphism $\tau: a \mapsto a b b a b, b \mapsto a b b$ generates a fixed point $\mathbf{x}_{\tau}$ having an infinite number of palindromes such that for all $\varphi \neq \operatorname{Id}$ verifying $\varphi\left(\mathbf{x}_{\tau}\right)=\mathbf{x}_{\tau}$, then $\varphi$ is not in the above extended class $\mathcal{P}$. However $\tau$ has a conjugate in class $\mathcal{P}$. Therefore, the initial question was reformulated in [Lab08].

Conjecture 1 (Hof, Knill, Simon; Blondin Massé, Labbé). Let $\mathbf{u}$ be the fixed point of a primitive morphism. Then, $\mathbf{u}$ is palindromic if and only if there exists a morphism $\varphi \neq \mathrm{Id}$ such that $\varphi(\mathbf{u})=\mathbf{u}$ and $\varphi$ has a conjugate in class $\mathcal{P}$.

Conjecture 1 has an important application on another question related to palindromic sequences with finite defect stated in [BMBGL08] also considered in [BPS11] for uniformly recurrent words. The conjecture states if the fixed point of a primitive morphism has a finite and positive defect, then it must be periodic. The question is still open for fixed point of primitive and injective morphisms since Bucci and Vaslet [BV12] noted that the fixed point of $a \mapsto a a b c a c b a, b \mapsto a a, c \mapsto a$ is not periodic and has defect 1 .

Partial results on Conjecture 1 have shown that morphisms preserving palindrome complexity must be in class $\mathcal{P}$, see [GJWZ09] for more details. The conjecture was answered positively for the periodic case in [ABCD03] and for the binary alphabet in [Tan07]. This last result is stronger than the conjecture, because it shows that the square of the morphism has a conjugate in class $\mathcal{P}$.

Theorem 2. [Tan07] Let $\varphi$ be a primitive morphism on a binary alphabet. Then, the fixed point of $\varphi$ is palindromic if and only if $\varphi^{2}$ has a conjugate in class $\mathcal{P}$.

It was also proven independently for uniform morphisms on the binary alphabet in [Lab08] using a different approach based on overlap of factors with a generalization to palindromes fixed by any antimorphism. In the uniform case for binary alphabet, it appears that conjugate morphisms are not needed.

Theorem 3. [Lab08] Let $\varphi$ be a uniform primitive morphism on a binary alphabet. Then, the fixed point of $\varphi$ is palindromic if and only if $\varphi$ or $\widetilde{\varphi}$ is in class $\mathcal{P}$ or the image of any letter by $\varphi^{2}$ is a palindrome (which means $\varphi^{2}$ is in class $\mathcal{P}$ ).

In this article, we show that a result like Theorem 2 or Theorem 3 is not possible beyond the binary alphabet. Indeed, we show the existence of a primitive morphism having a palindromic fixed point such that none of its power has a conjugate in class $\mathcal{P}$ (Lemma 16). We show even more by providing a counterexample to Conjecture 1 (Theorem 10). We manage to prove such a result by describing explicitly the stabilizer of a particular infinite sequence $\mathbf{x}$, i.e., the monoid of morphisms that fix $\mathbf{x}$, which is a hard problem in general. We use the properties of symmetric words, a notion related to class $\mathcal{P}$ morphisms. Definitions are in Section 2, the proof of the counterexample is in Section 3. 


\section{Definitions and notation}

\subsection{Combinatorics on words}

We borrow from M. Lothaire [Lot02] the basic terminology about words. In what follows, $\mathcal{A}$ is a finite alphabet whose elements are called letters. A word $w$ is a finite sequence of letters $w=w_{0} w_{1} \cdots w_{n-1}$ where $n \in \mathbb{N}$. The length of $w$ is $|w|=n$ and $w_{i}$ denotes its $i$-th letter. By convention the empty word is denoted $\varepsilon$ and its length is 0 . The set of all finite words over $\mathcal{A}$ is denoted by $\mathcal{A}^{*}$. Endowed with the concatenation, $\mathcal{A}^{*}$ is the free monoid generated by $\mathcal{A}$. The set of right infinite words is denoted by $\mathcal{A}^{\mathbb{N}}$ and the set of biinfinite words is $\mathcal{A}^{\mathbb{Z}}$. Given a word $w \in \mathcal{A}^{*} \cup \mathcal{A}^{\mathbb{N}}$, a factor $f$ of $w$ is a word $f \in \mathcal{A}^{*}$ satisfying $w=x f y$ for some $x \in \mathcal{A}^{*}$ and $y \in \mathcal{A}^{*} \cup \mathcal{A}^{\mathbb{N}}$. If $x=\varepsilon$ (resp. $y=\varepsilon$ ) then $f$ is called prefix (resp. suffix) of $w$. The set of all factors of $w$, called the language of $w$, is denoted by $\mathcal{L}(w)$. A word $w$ is primitive if it is not a power of another word, that is if $w=u^{p}$ for some word $u$ and integer $p$ then $w=u$ and $p=1$. Two words $u$ and $v$ are conjugate when there are words $x, y$ such that $u=x y$ and $v=y x$. A period of a word $w$ is an integer $p<|w|$ such that $w_{i}=w_{i+p}$, for all $i<|w|-p$. We recall the result of Fine and Wilf for doubly periodic words [Lot02, Theorem 8.1.4].

Lemma 4 (Fine and Wilf). Let $w$ be a word having period $p$ and $q$. If $|w| \geqslant p+q-$ $\operatorname{gcd}(p, q)$, then $\operatorname{gcd}(p, q)$ is also a period of $w$.

The reversal of $w=w_{0} w_{1} \cdots w_{n-1} \in \mathcal{A}^{n}$ is the word $\widetilde{w}=w_{n-1} w_{n-2} \cdots w_{0}$. A palindrome is a word $w$ such that $w=\widetilde{w}$. An infinite sequence $\mathbf{w}$ is palindromic if its language $\mathcal{L}(\mathbf{w})$ contains arbitrarily long palindromes.

The set of palindrome factors of a word $w$ is $\operatorname{Pal}(w)=\{p \in \mathcal{L}(w): \widetilde{p}=p\}$. It is known [DJP01] that $|\operatorname{Pal}(w)| \leqslant|w|+1$ for all finite word $w \in \mathcal{A}^{*}$. The defect $D(w)$ [BHNR04] of a finite word $w$ is the difference between the maximal number of palindromes that it may contain and the number of palindromes that it contains: $D(w)=|w|+1-|\operatorname{Pal}(w)|$. The defect of a infinite word $\mathbf{w}$ is $D(\mathbf{w})=\sup _{w \in \mathcal{L}(\mathbf{w})} D(w)$; it may be finite or infinite. A finite or infinite word $w$ is full or rich if $D(w)=0$.

\subsection{Symmetric words}

A word $w=w_{0} w_{1} \cdots w_{n-1}$ is symmetric if it is the product of two palindromes if and only if there exists an integer $a \in[0, n-1]$ such that

$$
w_{a-i}=w_{i} \quad \text { for all integer } i \in[0, n-1]
$$

where the indices are taken modulo $n$. We say that the integer $a$ is a point of symmetry of the word $w$. For example, the integer 6 is the unique point of symmetry of the word $w=a b c d c b a x y z z y x$. In the table below, we verify that $w_{6-i}=w_{i}$ for all integer $i \in[0,12]$ :

\begin{tabular}{c|ccccccccccccc}
$i$ & 0 & 1 & 2 & 3 & 4 & 5 & 6 & 7 & 8 & 9 & 10 & 11 & 12 \\
\hline$w_{i}$ & $a$ & $b$ & $c$ & $d$ & $c$ & $b$ & $a$ & $x$ & $y$ & $z$ & $z$ & $y$ & $x$ \\
$6-i$ & 6 & 5 & 4 & 3 & 2 & 1 & 0 & 12 & 11 & 10 & 9 & 8 & 7 \\
$w_{6-i}$ & $a$ & $b$ & $c$ & $d$ & $c$ & $b$ & $a$ & $x$ & $y$ & $z$ & $z$ & $y$ & $x$
\end{tabular}


The proofs of the following lemmas are easy.

Lemma 5. A finite $w$ word has a point of symmetry at $a$ if and only if $w=p q$ for some palindromes $p$ and $q$ such that $|p|=a+1$.

Lemma 6. Let $w$ and $w^{\prime}$ be two conjugate words, i.e., $u w=w^{\prime} u$ for some word $u$. If $w$ has a point of symmetry at $a$, then $w^{\prime}$ has a point of symmetry at $a+2|u| \bmod |w|$.

Lemma 7. If a finite word $w$ has two points of symmetry $a$ and $b$, then $\operatorname{gcd}(b-a,|w|)$ is a period of $w$. In particular, $w$ is not primitive.

Proof. Let $\mathbf{w}=\cdots w w \cdot w w w \cdots=\cdots w_{n-1} \cdot w_{0} w_{1} \cdots \in \mathcal{A}^{\mathbb{Z}}$ be the biinfinite word such that the index 0 of both $\mathbf{w}$ and $w$ coincide. The fact that $w$ has two points of symmetry $a$ and $b$ translates into the fact that $\mathbf{w}_{a-i}=\mathbf{w}_{b-i}=\mathbf{w}_{i}$ for all $i \in \mathbb{Z}$. Then $\mathbf{w}_{i}=\mathbf{w}_{a-i}=$ $\mathbf{w}_{b-(a-i)}=\mathbf{w}_{i+(b-a)}$ for all $i \in \mathbb{Z}$. Therefore, both $|w|$ and $b-a$ are periods of $\mathbf{w}$. We conclude by Lemma 4 that $\operatorname{gcd}(b-a,|w|)$ is a period of $\mathbf{w}$ and of $w$.

\subsection{Morphisms}

A morphism is a function $\varphi: \mathcal{A}^{*} \rightarrow \mathcal{A}^{*}$ compatible with concatenation, that is, such that $\varphi(u v)=\varphi(u) \varphi(v)$ for all $u, v \in \mathcal{A}^{*}$. The identity morphism on $\mathcal{A}$ is denoted by $\operatorname{Id}_{\mathcal{A}}$ or simply Id when the context is clear. A morphism $\varphi$ is primitive if there exists an integer $k$ such that for all $\alpha \in \mathcal{A}, \varphi^{k}(\alpha)$ contains each letter of $\mathcal{A}$. A morphism is called uniform when $|\varphi(\alpha)|=|\varphi(\beta)|$ for all letters $\alpha, \beta \in \mathcal{A}$. A morphism also extends in a natural way to a map over $\mathcal{A}^{\mathbb{N}}$. The set of sturmian morphisms is [MS93] the monoid generated by

$$
\left\{\begin{array}{rlrl}
a & \mapsto a b & a & \mapsto b a \\
b & \mapsto a
\end{array} \quad \begin{array}{rlrl}
a & \mapsto b
\end{array} \quad \begin{array}{ll}
a & \mapsto b
\end{array}\right\}
$$

A subset $X$ of the free monoid $\mathcal{A}^{*}$ is a code if there exists an injective morphism $\beta: \mathcal{B}^{*} \rightarrow$ $\mathcal{A}^{*}$ such that $X=\beta(\mathcal{B})$.

Recall from Lothaire [Lot02] (Section 2.3.4) that $\varphi$ is right conjugate of $\varphi^{\prime}$, or that $\varphi^{\prime}$ is left conjugate of $\varphi$, noted $\varphi \triangleleft \varphi^{\prime}$, if there exists $w \in \mathcal{A}^{*}$ such that

$$
\varphi(x) w=w \varphi^{\prime}(x), \quad \text { for all words } x \in \mathcal{A}^{*},
$$

or equivalently that $\varphi(\alpha) w=w \varphi^{\prime}(\alpha)$, for all letters $\alpha \in \mathcal{A}$. Clearly, this relation is not symmetric so that we say that two morphisms $\varphi$ and $\varphi^{\prime}$ are conjugate, if $\varphi \triangleleft \varphi^{\prime}$ or $\varphi^{\prime} \triangleleft \varphi$. It is easy to see that conjugacy of morphisms is an equivalence relation.

A morphism is erasing if the image of at least one of the letters is the empty word. If $\varphi$ is a nonerasing morphism, we define $\operatorname{Fst}(\varphi): \mathcal{A} \rightarrow \mathcal{A}$ to be the function defined by $\operatorname{Fst}(\varphi)(a)$ is the first letter of $\varphi(a)$. Similarly, let $\operatorname{Lst}(\varphi): \mathcal{A} \rightarrow \mathcal{A}$ be the function defined by $\operatorname{LsT}(\varphi)(a)$ is the last letter of $\varphi(a)$.

A morphism $\varphi$ is prolongable at $a$ if there is a letter $a$ such that $\varphi(a)=a w$ where $w$ is a nonempty word $w$. If $\varphi$ is prolongable at $a$, then

$$
\mathbf{w}=\operatorname{aw\varphi }(w) \varphi(\varphi(w)) \cdots \varphi^{n}(w) \cdots
$$


is a purely morphic word. It is also a fixed point of $\varphi$, i.e., $\varphi(\mathbf{w})=\mathbf{w}$. A morphic word is the image of a pure morphic word under a morphism.

The mirror-image of a morphism $\varphi$, denoted by $\widetilde{\varphi}$, is the morphism such that $\widetilde{\varphi}(\alpha)=$ $\widetilde{\varphi(\alpha)}$ for all $\alpha \in \mathcal{A}$. A morphism $\varphi$ is in class $\mathcal{P}$ if there exists a palindrome $p$ and for every $\alpha \in \mathcal{A}$ there exists a palindrome $q_{\alpha}$ such that $\varphi(\alpha)=p q_{\alpha}$. The mirror-image $\widetilde{\varphi}$ of a morphism $\varphi$ in class $\mathcal{P}$ is conjugate to $\varphi$. If a morphism has a conjugate in class $\mathcal{P}$, then the image of the letters are symmetric and they all share a common symmetry point which is quite demanding as we will see.

Lemma 8. If $\varphi$ has a conjugate in class $\mathcal{P}$, then there exists an integer $a \in \mathbb{N}$ such that for all $\alpha \in \mathcal{A}, \varphi(\alpha)$ is symmetric with a point of symmetry at a $\bmod |\varphi(\alpha)|$.

Proof. Let $\varphi^{\prime}$ in class $\mathcal{P}$ be the conjugate of $\varphi$. Let $p$ and $q_{\alpha}$ be the palindromes such that $\varphi^{\prime}(\alpha)=p q_{\alpha}$. Then for all letters $\alpha, \varphi^{\prime}(\alpha)$ has a point of symmetry at $|p|-1$ (Lemma 5). The result follows from Lemma 6 .

\subsection{Stabilizer}

The stabilizer of a right-infinite word $\mathbf{w}$ over a finite alphabet $\mathcal{A}$, denoted by $\operatorname{Stab}(\mathbf{w})$, is the monoid of morphisms $f: \mathcal{A}^{*} \rightarrow \mathcal{A}^{*}$ that satisfy $f(\mathbf{w})=\mathbf{w}$. Its unit element is the identity morphism. Words that have a cyclic stabilizer are called rigid. See [DK09, Kri08] for a discussion on the subject and [RS12] for a recently completed proof of the rigidity of words generated by sturmian morphisms.

Proposition 9. [Séé98, RS12] Sturmian words generated by sturmian morphisms are rigid.

\section{The counterexample}

Let $\mathcal{A}=\{a, b, c\}$ and $\gamma: \mathcal{A}^{*} \rightarrow \mathcal{A}^{*}$ be defined by

$$
\gamma: a \mapsto a c a, b \mapsto c a b, c \mapsto b
$$

Let $\mathbf{x}_{\gamma}$ be the fixed point of $\gamma$ starting with letter $a$ :

$$
\mathbf{x}_{\gamma}=\text { acabacacabacabacabacacabacabacacabacabac } \cdots
$$

In this section, we show that $\mathbf{x}_{\gamma}$ is a counterexample to Conjecture 1 . More precisely, we prove the following result.

Theorem 10. The fixed point $\mathbf{x}_{\gamma}$ of the primitive morphism $\gamma$ is palindromic, but none of the morphisms $\varphi \in \operatorname{Stab}\left(\mathbf{x}_{\gamma}\right) \backslash\{\operatorname{Id}\}$ has a conjugate in class $\mathcal{P}$. 


\section{1 $\mathrm{x}_{\gamma}$ is palindromic}

First, we show that the sequence $\mathbf{x}_{\gamma}$ is palindromic. Let $\left(p_{k}\right)_{k}$ be the sequence of finite words defined recursively by

$$
p_{-1}=a^{-1}, \quad p_{0}=b \quad \text { and } \quad p_{k+1}=\gamma\left(\operatorname{cap}_{k}\right) \quad \text { for each } \quad k>0 .
$$

The first terms are $p_{0}=b, p_{1}=$ bacacab and $p_{2}=$ bacacabacabacabacacab; these three words are palindromes. Also, the first letter of $p_{k}$ is $b$ for each $k \geqslant 0$. The next lemma shows that $\left(p_{k}\right)_{k}$ is a sequence of palindromes. Moreover, $p_{k}$ aca $p_{k-1}$ is not a palindrome only because it contains the centered factor $c a b$ or $b a c$ (the rest is perfectly symmetric with respect to the center).

Lemma 11. For all $k \geqslant 0$, the following statements are verified:

(i) $p_{k+1}=p_{k}$ aca $p_{k-1} a c a p_{k}$;

(ii) $p_{k}$ is a palindrome;

(iii) $p_{k-1}$ aca $p_{k}$ is not a palindrome.

Proof. (i) Notice that $p_{1}=p_{0} \cdot a c a \cdot p_{-1} \cdot a c a \cdot p_{0}=b a c a a^{-1} a c a b=b a c a c a b$ and $p_{2}=$ $p_{1} \cdot a c a \cdot p_{0} \cdot a c a \cdot p_{1}$. Suppose that $p_{k}=p_{k-1} \cdot a c a \cdot p_{k-2} \cdot a c a \cdot p_{k-1}$, for some $k \geqslant 2$. Then,

$$
\begin{aligned}
p_{k+1} & =\gamma\left(c a p_{k}\right) \\
& =\gamma\left(c a p_{k-1} \text { aca } p_{k-2} \text { aca } p_{k-1}\right) \\
& =\gamma\left(\operatorname{cap}_{k-1}\right) \gamma(a) \gamma\left(\operatorname{cap}_{k-2}\right) \gamma(a) \gamma\left(\operatorname{cap}_{k-1}\right) \\
& =p_{k} \text { aca } p_{k-1} \text { aca } p_{k} .
\end{aligned}
$$

(ii) We remark that $p_{0}$ and $p_{1}$ are palindromes. Also, from (i), if $p_{k-1}$ and $p_{k}$ are palindromes, then $p_{k+1}$ is a palindrome. Therefore $p_{k}$ is a palindrome for all $k \geqslant 0$.

(iii) First, $p_{-1} a c a p_{0}=a^{-1} a c a b=c a b$ and $p_{0} a c a p_{1}=$ bacabacacab are not palindromes. Suppose $p_{k-1} a c a p_{k}$ is not a palindrome for some $k \geqslant 1$. Then $p_{k}$ aca $p_{k+1}=$ $p_{k}$ aca $p_{k}$ aca $p_{k-1} a c a p_{k}$ is a palindrome if and only if the middle part $p_{k} a c a p_{k-1}$ is a palindrome. But, it is not being the reversal of $p_{k-1} a c a p_{k}$.

Lemma 12. $\mathbf{x}_{\gamma}$ is palindromic.

Proof. We show that for each $k \geqslant 0$, the word $p_{k}$ shown to be palindrome at Lemma 11 is included in $\mathcal{L}\left(\mathbf{x}_{\gamma}\right)$. Note that the first letter of $p_{k}$ is $b$ for each $k \geqslant 0$. Since each letter $b$ is preceded by the factor $c a$ in the language of $\mathbf{x}_{\gamma}$, we conclude that if $p_{k} \in \mathcal{L}\left(\mathbf{x}_{\gamma}\right)$, then $\operatorname{cap}_{k} \in \mathcal{L}\left(\mathbf{x}_{\gamma}\right)$ so that $p_{k+1}=\gamma\left(\operatorname{cap}_{k}\right) \in \mathcal{L}\left(\mathbf{x}_{\gamma}\right)$. Therefore, $p_{k} \in \mathcal{L}\left(\mathbf{x}_{\gamma}\right)$ for all $k \geqslant 0$.

Note that the previous two results are quite interesting. For a morphism $\varphi: \alpha \mapsto p q_{\alpha}$ in class $\mathcal{P}$, one shows that if $w$ is a palindrome, then $\varphi(w) p$ is also a palindrome. Here, the result says that prepending the non-palindromic word baca to $\gamma\left(p_{k}\right)$ makes it a new longer palindrome. This is a first indication that $\mathbf{x}_{\gamma}$ contains an infinite number of palindromes for a different reason than for well known fixed point of class $\mathcal{P}$ morphisms. 


\subsection{The stabilizer of $\mathrm{x}_{\gamma}$}

In this section, we describe exactly the stabilizer $\operatorname{Stab}\left(\mathbf{x}_{\gamma}\right)$ of the sequence $\mathbf{x}_{\gamma}$. This is a hard problem in general. Over a ternary alphabet, the monoid of morphisms generating a given infinite word by iteration can be infinitely generated [Kri08]. To achieve this, we show that the purely morphic sequence $\mathbf{x}_{\gamma}$ defined above is equal to the morphic sequence $\pi\left(\mathbf{w}_{\mu}\right)$ where

$$
\mu\left\{\begin{array}{l}
x \mapsto x y \\
y \mapsto x x y
\end{array}, \quad \pi\left\{\begin{array}{l}
x \mapsto a c \\
y \mapsto a b
\end{array}\right.\right.
$$

and $\mathbf{w}_{\mu}$ is the infinite fixed point of $\mu$ starting with letter $x$ :

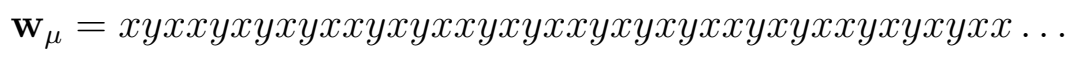

The finite prefix of $\mathbf{x}_{\gamma}$ shown earlier seems to indicate that the letter $a$ appears exactly at the even positions and that $b$ and $c$ letters appears at odd positions. This can be formulated by saying that $\mathbf{x}_{\gamma}$ is the image by the morphism $\pi$ of some infinite word over $\{x, y\}$. The next lemma shows that this infinite word is unique and is $\mathbf{w}_{\mu}$.

Lemma 13. We have $\gamma \circ \pi=\pi \circ \mu$. There exists a unique infinite word $\mathbf{w}$ such that $\mathbf{x}_{\gamma}=\pi(\mathbf{w})$. Moreover, $\mathbf{w}=\mathbf{w}_{\mu}$.

Proof. First, we verify that $\gamma \circ \pi=\pi \circ \mu$. It is sufficient to check it for letters:

$$
\begin{gathered}
\gamma(\pi(x))=\gamma(a c)=a c a b=\pi(x y)=\pi(\mu(x)), \\
\gamma(\pi(y))=\gamma(a b)=a c a c a b=\pi(x x y)=\pi(\mu(y)) .
\end{gathered}
$$

The word $\mathbf{x}_{\gamma}$ is an infinite product of two kinds of blocks of length 2 namely $a c$ and $a b$. Since $\{a c, a b\}$ is a code, there exists a unique infinite word $\mathbf{w}$ such that $\pi(\mathbf{w})=\mathbf{x}_{\gamma}$. But, using $\gamma \circ \pi=\pi \circ \mu$, we get

$$
\mathbf{x}_{\gamma}=\gamma\left(\mathbf{x}_{\gamma}\right)=\gamma(\pi(\mathbf{w}))=\pi(\mu(\mathbf{w})) .
$$

From the injectivity of $\pi$ we conclude that $\mathbf{w}=\mu(\mathbf{w})$ is a fixed point of $\mu$, i.e., $\mathbf{w}=\mathbf{w}_{\mu}$.

Lemma 14. Let $\varphi \in \operatorname{Stab}\left(\mathbf{x}_{\gamma}\right)$. Then, $|\varphi(a)|,|\varphi(b)|$ and $|\varphi(c)|$ are all odd or all even.

Proof. We have that $\varphi(a)$ is a prefix of $\mathbf{x}_{\gamma}$ and it must start with $a$. Therefore, $\varphi(b)$ and $\varphi(c)$ both do not end with $a$.

If $|\varphi(a)|$ is odd, then $\varphi(a)$ ends with letter $a, \varphi(c)$ does not start with letter $a$ so that $|\varphi(c)|$ is odd. Then $|\varphi(a c a)|$ is odd and hence $\varphi(b)$ does not start with letter $a$ from what we get that $|\varphi(b)|$ is odd as well.

If $|\varphi(a)|$ is even, then $\varphi(a)$ does no end with letter $a, \varphi(c)$ starts with letter $a$ so that $|\varphi(c)|$ is even. Then $|\varphi(a c a)|$ is even and hence $\varphi(b)$ starts with letter $a$ from what we get that $|\varphi(b)|$ is even as well.

The morphism $\mu$ is sturmian being factorized as $\mu=(x \mapsto x y, y \mapsto x) \circ(x \mapsto x, y \mapsto y x)$. Therefore, the sequence $\mathbf{w}_{\mu}$ is rigid. This is what allows us to describe exactly the stabilizer of $\mathbf{x}_{\gamma}$. 
Proposition 15. $\operatorname{Stab}\left(\mathbf{x}_{\gamma}\right)=\left\{\varphi\right.$ : there exists an integer $k$ such that $\left.\varphi \circ \pi=\gamma^{k} \circ \pi\right\}$.

Proof. Let $\varphi \in \operatorname{Stab}\left(\mathbf{x}_{\gamma}\right)$. From Lemma 14, $\varphi(a c)$ and $\varphi(a b)$ both have even length. Therefore, there exist words $u, v \in\{x, y\}^{*}$ such that $\varphi(\pi(x))=\varphi(a c)=\pi(u)$ and $\varphi(\pi(y))=\varphi(a b)=\pi(v)$. Since the image of the alphabet by $\pi$ is a code, the words $u$ and $v$ are uniquely determined. Hence, let $\sigma: x \mapsto u, y \mapsto v$. We have that $\varphi \circ \pi=\pi \circ \sigma$. From Lemma 13, $\mathbf{x}_{\gamma}=\pi\left(\mathbf{w}_{\mu}\right)$. We get

$$
\mathbf{x}_{\gamma}=\varphi\left(\mathbf{x}_{\gamma}\right)=\varphi\left(\pi\left(\mathbf{w}_{\mu}\right)\right)=\pi\left(\sigma\left(\mathbf{w}_{\mu}\right)\right) .
$$

From the injectivity of $\pi$, we get that $\mathbf{w}_{\mu}=\sigma\left(\mathbf{w}_{\mu}\right)$, that is $\sigma \in \operatorname{Stab}\left(\mathbf{w}_{\mu}\right)$. Since the fixed point $\mathbf{w}_{\mu}$ is sturmian and rigid, more precisely $\operatorname{Stab}\left(\mathbf{w}_{\mu}\right)=\langle\mu\rangle$, there exists an integer $k$ such that $\sigma=\mu^{k}$. Finally, from Lemma 13, we have $\pi \circ \mu^{k}=\gamma^{k} \circ \pi$. Thus, $\varphi \circ \pi=\pi \circ \sigma=\pi \circ \mu^{k}=\gamma^{k} \circ \pi$.

Reciprocally, suppose $\varphi$ is such that $\varphi \circ \pi=\gamma^{k} \circ \pi$. Then,

$$
\varphi\left(\mathbf{x}_{\gamma}\right)=\varphi\left(\pi\left(\mathbf{w}_{\mu}\right)\right)=\gamma^{k}\left(\pi\left(\mathbf{w}_{\mu}\right)\right)=\gamma^{k}\left(\mathbf{x}_{\gamma}\right)=\mathbf{x}_{\gamma} .
$$

Thus, $\varphi \in \operatorname{Stab}\left(\mathbf{x}_{\gamma}\right)$.

In other words, $\varphi \in \operatorname{Stab}\left(\mathbf{x}_{\gamma}\right)$ if and only if $\varphi \in S_{k}$ for some integer $k \geqslant 0$ where

$$
S_{k}=\left\{\varphi: \varphi(a b)=\gamma^{k}(a b) \text { and } \varphi(a c)=\gamma^{k}(a c)\right\} .
$$

The cardinality of the set $S_{k}$ is equal to the number of common prefixes of $\gamma^{k}(a b)$ and $\gamma^{k}(a c)$, i.e., Card $S_{k}=\left|\gamma^{k}(a)\right|+1$. The first values of this sequence can be computed easily:

\begin{tabular}{c|cccccccccc}
$k$ & 0 & 1 & 2 & 3 & 4 & 5 & 6 & 7 & 8 & 9 \\
\hline Card $S_{k}$ & 2 & 4 & 8 & 18 & 42 & 100 & 240 & 578 & 1394 & 3364
\end{tabular}

We have $\varphi \in S_{0}$ if and only if $\varphi=a \mapsto \varepsilon, b \mapsto a b, c \mapsto a c$ or $\varphi$ is the identity morphism. If $\varphi \in S_{1}$, then $\varphi$ is one of the following four morphisms:

$$
\gamma_{0}\left\{\begin{array}{l}
a \mapsto \varepsilon \\
b \mapsto a c a c a b \\
c \mapsto a c a b
\end{array} \quad, \quad \gamma_{1}\left\{\begin{array}{l}
a \mapsto a \\
b \mapsto c a c a b \\
c \mapsto c a b
\end{array} \quad, \quad \gamma_{2}\left\{\begin{array}{l}
a \mapsto a c \\
b \mapsto a c a b \\
c \mapsto a b
\end{array}, \quad \gamma=\gamma_{3}\left\{\begin{array}{l}
a \mapsto a c a \\
b \mapsto c a b \\
c \mapsto b
\end{array} .\right.\right.\right.\right.
$$

The indices for $\gamma$ are chosen according to the length of the image of $a$. If $\varphi \in S_{2}$, then $\varphi$ is one of the following eight morphisms:

$$
\begin{gathered}
\gamma_{0}^{2}\left\{\begin{array}{l}
a \mapsto \varepsilon \\
b \mapsto a c a b a c a b a c a c a b \\
c \mapsto a c a b a c a c a b
\end{array}, \gamma_{1}^{2}\left\{\begin{array}{l}
a \mapsto a \\
b \mapsto c a b a c a b a c a c a b \\
c \mapsto c a b a c a c a b
\end{array}, \gamma_{2} \gamma_{1}\left\{\begin{array}{l}
a \mapsto a c \\
b \mapsto a b a c a b a c a c a b \\
c \mapsto a b a c a c a b
\end{array}\right.\right.\right. \\
\gamma_{3} \gamma_{1}\left\{\begin{array}{l}
a \mapsto a c a \\
b \mapsto \text { bacabacacab } \\
c \mapsto \text { bacacab }
\end{array}, \gamma_{2}^{2}\left\{\begin{array}{l}
a \mapsto a c a b \\
b \mapsto \text { acabacacab } \\
c \mapsto \text { acacab }
\end{array}, \gamma_{1} \gamma_{3}\left\{\begin{array}{l}
a \mapsto a c a b a \\
b \mapsto c a b a c a c a b \\
c \mapsto c a c a b
\end{array}\right.\right.\right.
\end{gathered}
$$




$$
\gamma_{2} \gamma_{3}\left\{\begin{array}{l}
a \mapsto a c a b a c \\
b \mapsto a b a c a c a b \\
c \mapsto a c a b
\end{array}, \gamma_{3}^{2}\left\{\begin{array}{l}
a \mapsto \text { acabaca } \\
b \mapsto b a c a c a b \\
c \mapsto c a b
\end{array} .\right.\right.
$$

In this case, we have $\left(S_{1}\right)^{2}=S_{2}$. However, this is not true in general as $\left(S_{1}\right)^{3} \subsetneq S_{3}$. In other words, $S_{1}=\left\{\gamma_{0}, \gamma_{1}, \gamma_{2}, \gamma_{3}\right\}$ do not form a set of generators for $\operatorname{Stab}\left(\mathbf{x}_{\gamma}\right)$. In fact, Card $\left(S_{1}\right)^{3}=16$ but Card $S_{3}=18$. The two elements of $S_{3} \backslash\left(S_{1}\right)^{3}$ are

$$
\begin{aligned}
& a \mapsto \text { acabacac, } b \mapsto \text { abacabacacabacabacabacacab, } c \mapsto \text { abacabacabacacab, } \\
& a \mapsto \text { acabacaca, } b \mapsto \text { bacabacacabacabacabacacab, } c \mapsto \text { bacabacabacacab. }
\end{aligned}
$$

\subsection{Proof of Theorem 10}

Before proving the theorem, we show that the powers of $\gamma$ itself do not have any conjugate in class $\mathcal{P}$. This implies that results like Theorem 2 or Theorem 3 are not possible beyond the binary alphabet.

Lemma 16. For all $k \geqslant 1, \gamma^{k}$ does not have any conjugate in class $\mathcal{P}$.

In the following proof, if $\varphi$ is a morphism on $\mathcal{A}^{*}$ with $\mathcal{A}=\{a, b, c\}$, we represent the function $f=\operatorname{Fst}(\varphi): \mathcal{A} \rightarrow \mathcal{A}$ as an ordered tuple $(f(a), f(b), f(c))$ and similarly for $\operatorname{LsT}(\varphi): \mathcal{A} \rightarrow \mathcal{A}$.

Proof. We have that $\operatorname{FsT}\left(\gamma^{k}\right)=(a, b, c)$ for all even $k$ and $\operatorname{Fst}\left(\gamma^{k}\right)=(a, c, b)$ for all odd $k$. Also $\operatorname{LsT}\left(\gamma^{k}\right)=(a, b, b)$ for all $k$. Since none of these functions is constant, $\gamma^{k}$ does not have conjugates other than itself. For the same reason, the image of each letter by $\gamma^{k}$ must be a palindrome in order to be in class $\mathcal{P}$. But it is not the case $\operatorname{since} \operatorname{FsT}\left(\gamma^{k}\right) \neq \operatorname{LST}\left(\gamma^{k}\right)$ for all $k$.

We show that the words $\gamma^{k}(a b)$ and $\gamma^{k}(a c)$ are symmetric with distinct points of symmetry.

Lemma 17. We have

(i) $\gamma^{k}(a b)=$ aca $p_{k-2}$ aca $p_{k-1}$ is a symmetric word with a point of symmetry at $\left|p_{k-2}\right|+5$ for all $k \geqslant 2$,

(ii) $\gamma^{k}(a c)=a c a p_{k-1}$ is a symmetric word with a point of symmetry at 2 for all $k \geqslant 1$,

Proof. (i) We have

$$
\gamma^{2}(a b)=a c a b a c a b a c a c a b=a c a p_{0} a c a p_{1} .
$$

Suppose $\gamma^{k}(a b)=a c a p_{k-2}$ aca $p_{k-1}$ for some $k \geqslant 2$. Then

$$
\begin{aligned}
\gamma^{k+1}(a b)=\gamma\left(\gamma^{k}(a b)\right) & =\gamma\left(a c a p_{k-2} a c a p_{k-1}\right) \\
& =\gamma(a) \gamma\left(c a p_{k-2}\right) \gamma(a) \gamma\left(c a p_{k-1}\right)=a c a p_{k-1} a c a p_{k}
\end{aligned}
$$

(ii) We verify $\gamma(a c)=a c a b=a c a p_{0}$. Suppose $\gamma^{k}(a c)=a c a p_{k-1}$ for some $k \geqslant 1$. Then

$$
\gamma^{k+1}(a c)=\gamma\left(\gamma^{k}(a c)\right)=\gamma\left(\operatorname{aca~}_{k-1}\right)=\gamma(a) \gamma\left(\operatorname{cap}_{k-1}\right)=a c a p_{k} .
$$


We also need the property that the words $\gamma^{k}(a b)$ and $\gamma^{k}(a c)$ are primitive. This is a consequence of the more general following result showing that $\mu$ preserves primitivity as well as $\gamma$ under some extra conditions.

Lemma 18. We have

(i) for all $w \in\{x, y\}^{*}, \mu(w)$ is primitive if and only if $w$ is primitive,

(ii) for all $w \in \pi\left(\{x, y\}^{*}\right), \gamma(w)$ is primitive if and only if $w$ is primitive,

Proof. (i) ( $\Longrightarrow$ ) If $w$ is not primitive, it can be written as $w=u^{p}$ for some word $u$ and integer $p \geqslant 2$. Therefore, $\mu(w)=\mu\left(u^{p}\right)=\mu(u)^{p}$ is not primitive. ( $\left.\Longleftarrow\right)$ If $\mu(w)$ is not primitive, there exist a word $u$ and an integer $p \geqslant 2$ such that $\mu(w)=u^{p}$. Then $u$ starts with the letter $x$ and ends with letter $y$. Therefore, $u$ can be desubstituted uniquely as $u=\mu(v)$ for some word $v$. We get $\mu(w)=u^{p}=\mu(v)^{p}=\mu\left(v^{p}\right)$. From the fact that the letter images of $\mu$ is a prefix code, we get that $w=v^{p}$ and $w$ is not primitive.

(ii) $(\Longrightarrow)$ The proof is the same as in (i). ( a word $u$ and an integer $p \geqslant 2$ such that $\gamma(w)=u^{p}$. The last letter of $u$ must be $b$ and the first letter of $u$ must be $a$. Hence, $u$ can be written as the image under $\gamma$ of some word $v$. The word $v$ is unique because the images of letters by $\gamma$ is a prefix code. We get $\gamma(w)=u^{p}=\gamma(v)^{p}=\gamma\left(v^{p}\right)$ and thus $w=v^{p}$ because $\gamma$ forms a code. Then, $w$ is not primitive.

We have now gathered enough information to prove the main result.

Proposition 19. If $\varphi \in \operatorname{Stab}\left(\mathbf{x}_{\gamma}\right) \backslash\{\operatorname{Id}\}$, then $\varphi$ does not have a conjugate in class $\mathcal{P}$.

Proof. Let $\varphi \in \operatorname{Stab}\left(\mathbf{x}_{\gamma}\right)$. From Proposition 15, we have that $\varphi(a b)=\gamma^{k}(a b)$ and $\varphi(a c)=$ $\gamma^{k}(a c)$ for some integer $k$. We may suppose that $k \geqslant 1$ since $\varphi$ is not the identity. Suppose by contradiction that $\varphi$ has a conjugate in class $\mathcal{P}$. We may assume that $k \geqslant 2$ since we may check that none of $\gamma_{0}, \gamma_{1}, \gamma_{2}, \gamma_{3}$ defined above have a conjugate in class $\mathcal{P}$. This means that there exist a word $w$ and palindromes $q, p_{a}, p_{b}$ and $p_{c}$ such that

$$
\begin{aligned}
& w p_{a} q=\varphi(a) w, \quad p_{a} q w=w \varphi(a), \\
& w p_{b} q=\varphi(b) w, \quad \text { or } \quad p_{b} q w=w \varphi(b) \text {, } \\
& w p_{c} q=\varphi(c) w, \quad \quad p_{c} q w=w \varphi(c) \text {. }
\end{aligned}
$$

Therefore $\varphi(a b)$ and $\varphi(a c)$ are symmetric words with the same axis of symmetry. Indeed, from the equations above we get that $\varphi(a b)$ and $\varphi(a c)$ are conjugate to symmetric words having a point of symmetry at $\left|p_{a}\right|-1$ (Lemma 5):

$$
\begin{array}{ll}
\varphi(a b) \cdot w=w \cdot p_{a} q p_{b} q, \\
\varphi(a c) \cdot w=w \cdot p_{a} q p_{c} q .
\end{array} \quad \text { or } \quad \begin{aligned}
& w \cdot \varphi(a b)=p_{a} q p_{b} q \cdot w \\
& w \cdot \varphi(a c)=p_{a} q p_{c} q \cdot w .
\end{aligned}
$$

From Lemma 6, $\varphi(a b)$ and $\varphi(a c)$ both have a point of symmetry at $A=\left|p_{a}\right|-1+2|w|$ or at $A=\left|p_{a}\right|-1-2|w|$. From Lemma 17, $\varphi(a b)$ already has a point of symmetry at $\left|p_{k-2}\right|+5$. If $A \neq\left|p_{k-2}\right|+5$, then $\varphi(a b)$ has two distinct points of symmetry. Then, $\varphi(a b)$ 
is periodic with period $g$ where $g$ is a divisor of $|\varphi(a b)|$ (Lemma 7). Therefore, $\varphi(a b)$ is not primitive which is a contradiction. From Lemma 18, $\varphi(a b)$ is primitive because from the beginning $a b$ is a primitive word.

If $A=\left|p_{k-2}\right|+5$, then 2 and $A$ are two distinct points of symmetry of $\varphi(a c)$. We can get a contradiction using a primitivity argument as above, but also using an argument based on palindromes. We have

$$
\varphi(a c)=a c a p_{k-1}=a c a p_{k-2} a c a p_{k-3} a c a p_{k-2}
$$

from Lemma 17. A point of symmetry at $A=\left|p_{k-2}\right|+5$ implies that $p_{k-3} a c a p_{k-2}$ is a palindrome which is a contradiction with Lemma 11 (iii). We conclude that none of the conjugate of $\varphi$ are in class $\mathcal{P}$.

Proof of Theorem 10. Follows from Lemma 12 and Proposition 19.

\section{Conclusion}

We have seen that the fixed point $\mathbf{x}_{\gamma}$ of $\gamma$ is not rigid. It is still open to show that its stabilizer is not finitely generated. We have shown that the infinite sequence $\mathbf{x}_{\gamma}$ is palindromic but none of the morphisms that stabilizes it have a conjugate in class $\mathcal{P}$. This is thus a counterexample to Conjecture 1 which was considered for a long time as the interpretation of the question of Hof, Knill and Simon.

The characteristic polynomial of the incidence matrix of $\gamma$ is not irreducible as it factorizes as $(x-1) \cdot\left(x^{2}-2 x-1\right)$. Note that $x^{2}-2 x-1$ is the characteristic polynomial of the incidence matrix of $\mu$. It is still an open question whether there exists a counterexample to Conjecture 1 such that the characteristic polynomial is irreducible, or if there exists a counterexample that is not the image on a larger alphabet of a purely morphic word.

Note that $\gamma_{0}: a \mapsto \varepsilon, b \mapsto a c a c a b, c \mapsto a c a b$ is almost in class $\mathcal{P}$. This leads to think that the question of Hof, Knill and Simon could be fixed once more by including erasing morphisms such that their nonerasing part has a conjugate in class $\mathcal{P}$. More investigations need to be done here to find a proper statement of the original question of Hof, Knill and Simon.

Recent work [HVZ13] was done in that direction motivated by our counterexample suggesting that the good interpretation of the question of Hof, Knill and Simon should be that if $\mathbf{u}$ is a primitive morphic word and $\mathbf{u}$ is palindromic, then there exist two morphisms $\varphi$ and $\varphi^{\prime}$ such that $\mathbf{u}=\varphi(\mathbf{v}), \varphi^{\prime}(\mathbf{v})=\mathbf{v}$ and both $\varphi$ and $\varphi^{\prime}$ have a conjugate in class $\mathcal{P}$. They remarked that this property holds for $\mathbf{x}_{\gamma}$ since $\pi$ and $\mu$ have a conjugate in class $\mathcal{P}$. They show that this statement holds for sequences $\mathbf{u}$ that are primitive morphic word with finite defect.

\section{Acknowledgements}

The author is thankful to the anonymous referee whose suggestions improved considerably the presentation. 


\section{References}

[ABCD03] J.-P. Allouche, M. Baake, J. Cassaigne, and D. Damanik. Palindrome complexity. Theoret. Comput. Sci., 292(1):9-31, 2003.

[BHNR04] S. Brlek, S. Hamel, M. Nivat, and C. Reutenauer. On the palindromic complexity of infinite words. Int. J. Found. Comput. Sci., 15(2):293-306, 2004.

[BMBGL08] A. Blondin-Massé, S. Brlek, A. Garon, and S. Labbé. Combinatorial properties of $f$-palindromes in the Thue-Morse sequence. Pure Math. Appl. (PU.M.A.), 19(2-3):39-52, 2008.

[BPS11] L. Balková, E. Pelantová, and Š. Starosta. Infinite words with finite defect. Adv. in Appl. Math., 47(3):562-574, 2011.

[BV12] M. Bucci and E. Vaslet. Palindromic defect of pure morphic aperiodic words. 14th Mons Days of Theoretical Computer Science, 2012 (talk).

[DJP01] X. Droubay, J. Justin, and G. Pirillo. Episturmian words and some constructions of de Luca and Rauzy. Theoret. Comput. Sci., 255(1-2):539-553, 2001.

[DK09] V. Diekert and D. Krieger. Some remarks about stabilizers. Theoret. Comput. Sci., 410(30-32):2935-2946, 2009.

[GJWZ09] A. Glen, J. Justin, S. Widmer, and L. Q. Zamboni. Palindromic richness. European J. Combin., 30(2):510-531, 2009.

[HKS95] A. Hof, O. Knill, and B. Simon. Singular continuous spectrum for palindromic Schrödinger operators. Comm. Math. Phys., 174(1):149-159, 1995.

[HVZ13] T. Harju, J. Vesti, and L. Q. Zamboni. On a remark of Hof, Knill and Simon on palindromic substitutive systems. arXiv:1311.0185v1, Nov 12013.

[Kri08] D. Krieger. On stabilizers of infinite words. Theoret. Comput. Sci., 400(1-3):169181, 2008.

[Lab08] S. Labbé. Propriétés combinatoires des $f$-palindromes. Mémoire de maîtrise, Université du Québec à Montréal, Montréal, 2008. M10615.

[Lot02] M. Lothaire. Algebraic combinatorics on words, volume 90 of Encyclopedia of Mathematics and its Applications. Cambridge University Press, Cambridge, 2002.

[MS93] F. Mignosi and P. Séébold. Morphismes sturmiens et règles de Rauzy. J. Théor. Nombres Bordeaux, 5(2):221-233, 1993.

[RS12] G. Richomme and P. Séébold. Completing a combinatorial proof of the rigidity of Sturmian words generated by morphisms. Theoret. Comput. Sci., 428:92-97, 2012.

[Séé98] P. Séébold. On the conjugation of standard morphisms. Theoret. Comput. Sci., 195(1):91-109, 1998. Mathematical foundations of computer science (Cracow, 1996).

[Tan07] B. Tan. Mirror substitutions and palindromic sequences. Theoret. Comput. Sci., 389(1-2):118-124, 2007. 